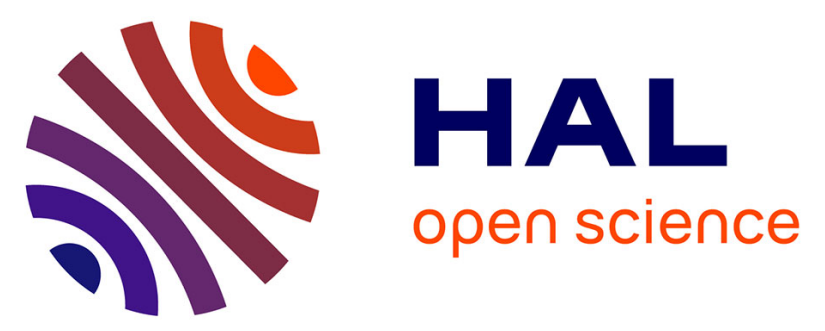

\title{
Real-time Muscle Deformation via Decoupled Modeling of Solid and Muscle Fiber Mechanics
}

Mohamed Yacine Berranen, Mitsuhiro Hayashibe, David Guiraud, Benjamin Gilles

\section{- To cite this version:}

Mohamed Yacine Berranen, Mitsuhiro Hayashibe, David Guiraud, Benjamin Gilles. Real-time Muscle Deformation via Decoupled Modeling of Solid and Muscle Fiber Mechanics. MICCAI: Medical Image Computing and Computer-Assisted Intervention, Sep 2014, Boston, MA, United States. pp.65-72, 10.1007/978-3-319-10470-6_9 . lirmm-01102481

\section{HAL Id: lirmm-01102481 https://hal-lirmm.ccsd.cnrs.fr/lirmm-01102481}

Submitted on 12 Jan 2015

HAL is a multi-disciplinary open access archive for the deposit and dissemination of scientific research documents, whether they are published or not. The documents may come from teaching and research institutions in France or abroad, or from public or private research centers.
L'archive ouverte pluridisciplinaire HAL, est destinée au dépôt et à la diffusion de documents scientifiques de niveau recherche, publiés ou non, émanant des établissements d'enseignement et de recherche français ou étrangers, des laboratoires publics ou privés. 


\title{
Real-time Muscle Deformation via Decoupled Modeling of Solid and Muscle Fiber Mechanics
}

\author{
Yacine Berranen, Mitsuhiro Hayashibe, David Guiraud and Benjamin Gilles \\ INRIA DEMAR Project, LIRMM CNRS and University of Montpellier Sud de \\ France. $\{$ berranen, hayashibe, guiraud, gilles\}@lirmm.fr
}

\begin{abstract}
This paper presents a novel approach for simulating 3D muscle deformations with complex architectures. The approach consists in choosing the best model formulation in terms of computation cost and accuracy, that mixes a volumetric tissue model based on finite element method (3D FEM), a muscle fiber model (Hill contractile 1D element) and a membrane model accounting for aponeurosis tissue (2D FEM). The separate models are mechanically binded using barycentric embeddings. Our approach allows the computation of several fiber directions in one coarse finite element, and thus, strongly decreases the required finite element resolution to predict muscle deformation during contraction. Using surface registration, fibers tracks of specific architecture can be transferred from a template to subject morphology, and then simulated. As a case study, three different architectures are simulated and compared to their equivalent one dimensional Hill wire model simulations.
\end{abstract}

\section{Introduction}

To understand the musculoskeletal mechanical behaviors, in particular muscle strain injuries, or predict a post-surgical effect, accurate biomechanical with functional modelings are mandatory. It gives biomechanicians, orthopedists and surgeons, a way to evaluate the impact of a therapy on both the muscle mechanical structure and its force development. Computational muscle models provide a powerful tool to simulate, visualize and analyze results. However, the research purposes regarding simulations are often drastically different. While some focus on the study of limb's movements, others observe muscle deformations within very small volumes of few cubic millimeters. Therefore, muscle modeling resulted in completely different approaches.

Many developed models [1] and frameworks [2], generally based on Hill type model for the force generation, consider the muscle as a 1D wire-segment with geometrical constraints of surrounding materials, in order to provide accurate, real-time and interactive simulations of the induced movements. However, 1D muscle wire models are based on lumped-parameters and may not be suitable to represent the behavior of muscles with complex fiber architecture for detailed analysis [3]. Moreover, they require many assumptions and do not provide information about muscle volumetric deformations during contraction.

To overcome lumped-parameters models limitations, muscle is simulated in its 
entire volume derived from MRI acquisitions of specific subjects, by detailing its fascicle architecture and tendons configuration. The classical approach of 3D models relies on Finite Element Method (FEM). Several formulations of continuum models of skeletal muscles have been developed. The standard deformationcoupling invariants were used to simulate contractile skeletal muscle $[4,5]$. A new set of invariants was proposed [6], it allows for representation of muscle tissue resistance to along-fiber shear and cross-fiber shear. Another formulation of the strain energy was presented using only isotropic strain invariants [7], separating anisotropic muscle tissue response from the isotropic strain energy stored in the fibers.

One can show that linear springs embedded into a linear finite element (triangle or tetrahedra) can be equivalently modeled using an anisotropic material law. However, this is not possible when non-linearities are introduced in finite element shape functions (leading to inhomogeneous deformations within the finite element), or in spring material law (non-linear muscle force/length relationship). One finite element can thus only account for one average fiber direction. Therefore, for the purely FEM methods referenced above, the FE resolution has to fit the fiber architecture complexity. In [6], to represent the biceps brachii, approximately 20,000 hexahedral elements are used to achieve the simulation. This makes real-time simulation of musculoskeletal movement involving several muscles, impractical.

In this paper, we present a novel method to simulate 3D muscles with complex architectures in real-time using a combined models formulation. We propose to mechanically bind three models with separate discretizations using barycentric embeddings. Many fiber directions are computed per finite element, that leads to muscle representation with a few number of finite elements and decreases simulation computation time. We demonstrate that our combined modeling fits validated 1D Hill models in term of produced force and allows prediction of muscle deformations and stiffness with close to real-time computations. Moreover, We show how fibers tracks of specific architectures can be transferred from a template to the real subject morphology extracted from medical images. The paper introduces the new method, then shows results and ends with a discussion.

\section{Methods}

Muscle material behavior can be decomposed into an isotropic part (tissue matrix) and anisotropic part (fibers and membranes). Instead of combining related models within a single strain energy density expression, and subsequently discretize it with fine finite elements, we use several models of different discretizations that match the complexity of the underlying behavior (see Fig. 1). This is equivalent to have many integration points per element, to account for behavior non-uniformity. Forces computed from these models are blended at a master level (kinematic model) using the barycentric embedding technique (Sec. 2.1). Muscle deformation being relatively smooth, we use a kinematic model with only a few degrees of freedom (nodes of a coarse embedding grid). This allows fast 
time integration. However, as the deformation emerges from a complex network of orientation-changing fibers (Sec. 2.2) with non-linear behavior (Sec. 2.3), we model the internal architecture with detailed discrete curves. The isotropic, lowfrequency behavior of the tissue matrix is integrated at the coarse level using hexahedral elements. The aponeurosis which is a stiff membranous layer where fibers are inserted is discretized using triangle FEM.

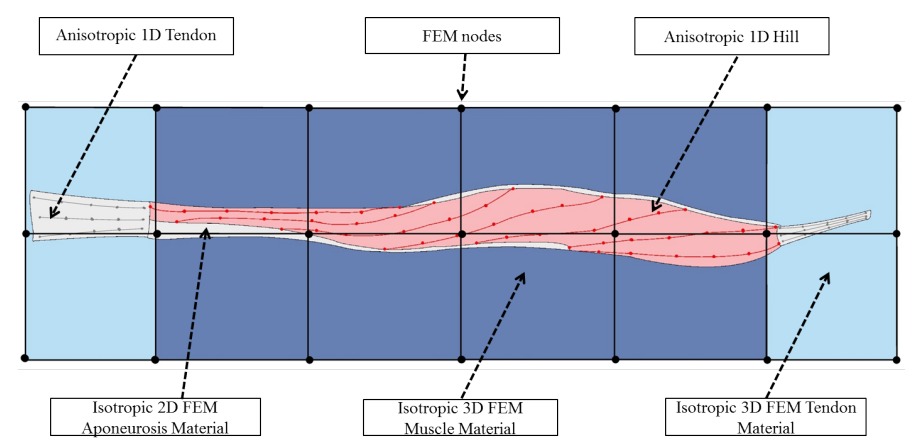

Fig. 1. Muscle multi-model scheme : The different models are linked via barycentric embeddings. This approach strongly decreases the required finite element resolution to predict muscle deformation during contraction

\subsection{Barycentric Embeddings}

Barycentric embedding, that we briefly review here, is a common approach in multi-model frameworks, where hard kinematical and mechanical coupling is necessary [8]. Let $J_{p}$ be the function used to map the $3 \mathrm{D}$ positions $x_{m}$ of a master node to the positions $x_{s}$ of a slave :

$$
x_{s}=J_{p}\left(x_{m}\right)
$$

The velocities are mapped in the same way, where the Jacobian $J_{v}=\frac{\partial x_{s}}{\partial x_{m}}$ computes the linear relation between master and slave velocities:

$$
v_{s}=J_{v} \cdot v_{m}
$$

In our case, the mapping is linear. Operators $J_{p}$ and $J_{v}$ are the same $(J)$, and contains the barycentric coordinates of the slave vertices in their corresponding FEM element. The master positions and the velocities are propagated down to the slaves. Inversely, the forces are propagated bottom-up to the independent DOFs of the master, where Newton's law $f=M a$ is applied. Given forces $f_{s}$ produced by a slave model, we can compute the equivalent forces $J^{T} f_{s}$ based on the principle of virtual work, and accumulate slaves contributions at the master level:

$$
f_{m} \longleftarrow f_{m}+J^{T} f_{s}
$$




\section{$2.2 \quad$ Fiber Geometry Specification}

The chosen approach for approximating the muscle architectures depending on our given surface, is inspired from the one proposed by [3], where solid hexahedral cuboidal templates that consist of geometrical set of control points, are linearly interpolated using rational Bezier spline curves to discretize the fiber field of a given fiber architecture. The cuboidal template undergoes a one-to-one morphing on a muscle mesh geometry, to generate the target discretized equally spaced points $\left(X_{\text {pnt }}^{\text {target }}\right)$. As we have separately modeled the FEM model from Hill contractile element models, we free ourselves from the traditional fiber direction computation, that consists on the tangent of the curve in each target point $\left(X_{\text {pnt }}^{\text {target }}\right)$, and requires several steps. There is no need to involve fiber directions in each FEM elements, all is managed through embedding.

\subsection{Constitutive Models}

Contractile Element Model We connect the points $\left(X_{\text {pnt }}^{\text {target }}\right)$ by Hill contractile element that generates non-linear forces with respect to the deformation (eq. 4). They are function of $a(t)$ the activation from 0 to 1 that we consider uniform over muscle volume.

$$
F_{c e}(t)=a(t) f_{l}\left(\varepsilon_{c}\right) f_{v}\left(\dot{\varepsilon}_{c}\right) F_{0}^{m} / N b f
$$

$F_{c e}(t)$ is the contractile element force between the two adjacent nodes of a fiber segment. $\varepsilon_{c}$ is the strain of the contractile element (muscle model), related to current and optimal fiber length $L(t)$ and $L c 0$ through: $\varepsilon_{c}=L(t) / L c 0-1 . F_{0}^{m}$ maximum isometric force of the whole muscle. $N b f$ number of individual fibers depending on origin-insertion surface in $\left(\mathrm{cm}^{2}\right)$ multiplied by the fiber density $\rho\left(\right.$ fibers $\left./ \mathrm{cm}^{2}\right) . f_{l}\left(\varepsilon_{c}\right)$ represents force-length relationship approximated by a Gaussian distribution around $L c 0$, where $b$ is a constant material parameter (set to 0.5). Anisotropic passive properties along the fiber are neglected because isometric concentric contractions are studied in this paper. However, 1D passive properties can be easily embedded in our contractile element model to reinforce fibers and simulate eccentric contractions. $f_{v}\left(\dot{\varepsilon}_{c}\right)$ is the force-velocity relationship that depends on $\dot{\varepsilon}_{c}$ (strain velocity), $V_{\max }$ the maximum velocity of individual muscle fiber (set to $\left.5\left(1+a(t) \cdot f_{l}\left(\varepsilon_{c}\right)\right)\right) / N b f$ ) and $V_{s h}$ a constant adimensional material parameter (set to 0.3 ) [9].

$$
f_{l}\left(\varepsilon_{c}\right)=\exp \left\{-\left(\frac{\varepsilon_{c}}{b}\right)^{2}\right\} \quad f_{v}\left(\dot{\varepsilon}_{c}\right)=\frac{V_{s h}\left(V_{\max }+L_{c 0} \dot{\varepsilon}_{c}\right)}{V_{s h} V_{\max }-L_{c 0} \dot{\varepsilon}_{c}}
$$

Isotropic Tissue Model As shown in [7], the passive isotropic behavior of muscle-tendon units can be well approximated by a quasi-incompressible 2nd order polynomial material law:

$$
W_{\text {matrix }}=\sum_{i+j=1}^{2} C_{i j}\left(\bar{I}_{1}-3\right)^{i}\left(\bar{I}_{2}-3\right)^{j}+\frac{K}{2}(J-1)^{2}
$$



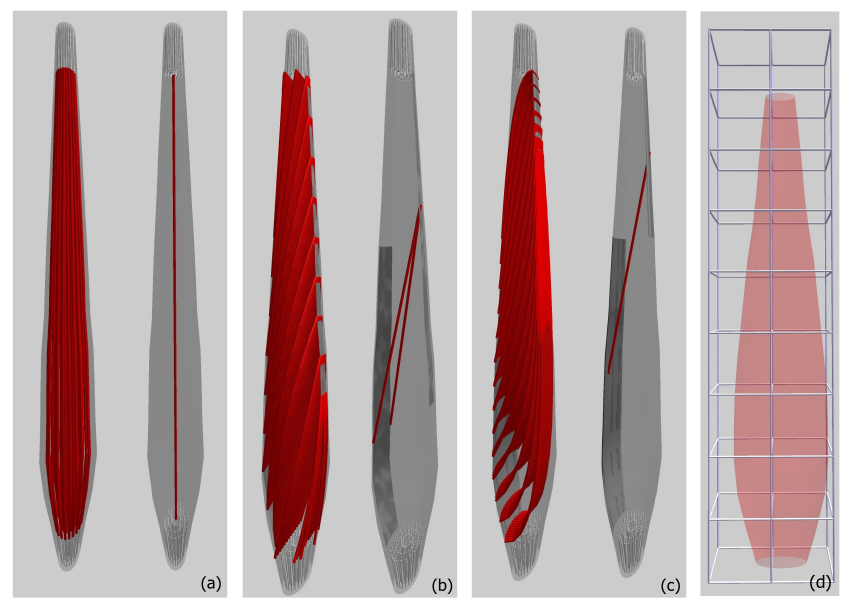

Fig. 2. (a,b,c) : geometrical approximations of complex fiber architectures (Parallel, Bipennate, Pennate) and their equivalent 1D Hill wire model; (d) : coarse FEM hexahedron geometry generated by surface rasterization.

where $W_{\text {matrix }}$ is the strain energy density, $\bar{I}_{1}$ and $\bar{I}_{2}$ are deviatoric invariants of the right Cauchy deformation tensor. We assign different material parameters to FEM hexaedra, depending on the underlying tissue. In $\mathrm{N} / \mathrm{cm}^{2}: C_{10}=6.43$, $C_{01}=-3.81, C_{20}=0.94, C_{02}=0.0005, C_{11}=-0.0043, K=5 E 4$ for muscle elements; $C_{10}=30, C_{20}=80, C_{30}=800, K=2 E 5$ for tendon elements [7].

Aponeurosis Model Aponeurosis are stiff membranes where muscle fibers are attached to. The passive isotropic stiffness described above is reinforced along these membranes using triangle FE meshes, embedded into the master hexahedral grid. We use a Hookean material with a Young modulus of $200 \mathrm{kPa}$ [7]. The thickness of the membrane is set to $1 \mathrm{~mm}$. Similarly, we reinforce tendon material stiffness along fibers using embedded 1D springs (Young modulus=200kPa).

\section{$3 \quad$ Results}

\subsection{Typical Muscle Model}

We define a typical muscle surface mesh as a generalized cylinder in Matlab, embedded in a coarse hexahedral FEM grid generated by surface rasterization Fig. 2. d. The exact volume of underlying material is assigned to FEM Gauss points to avoid energy integration in empty parts of hexahedra. The cuboidal templates of three different architectures (see Fig. 2) are morphed to the muscle surface mesh . To compare the three muscle architectures in the same conditions, we computed the fiber insertion surface mean value of each architecture. We fixed 

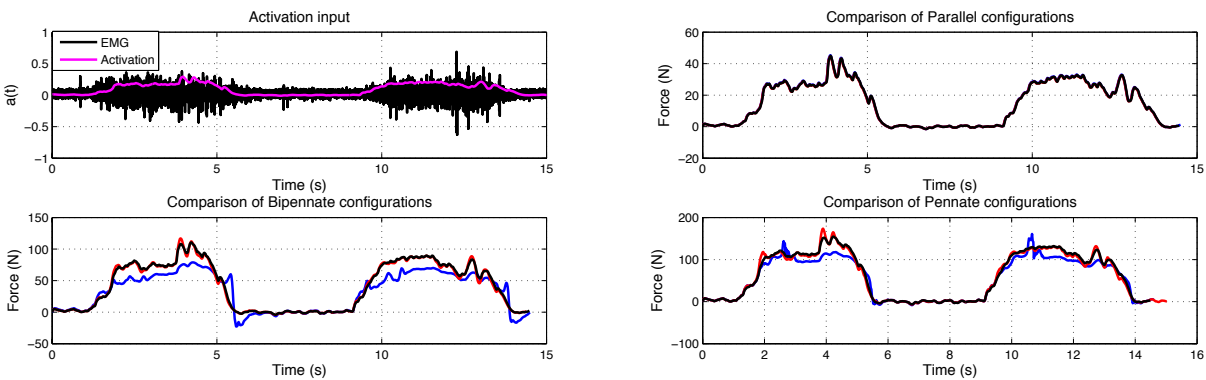

Fig. 3. Comparison of longitudinal force production performance in 3 different muscle fiber architectures. The blue line represents the result with classical Hill wire model while the red and black lines indicate the case with the proposed volumetric modeling in coarse FEM resolution and fine FEM resolution, respectively. Total forces are measured on tendon extremities while varying muscle activation in time.

the number of bipennate fibers to 150 and its $F_{0}^{m}$ to $1000 N$. We computed the fiber density $\rho$ by dividing the number of fibers $N b f(150)$ by the bipennate fiber insertion surface $\left(13.947 \mathrm{~cm}^{2}\right)$. Keeping the same obtained $\rho$ of $10.75\left(1 / \mathrm{cm}^{2}\right)$, we computed $\mathrm{Nbf}$ and $F_{0}^{m}$ of parallel and pennate architectures, for respectively given surfaces of $\left(2.232 \mathrm{~cm}^{2}\right)$ and $\left(17.496 \mathrm{~cm}^{2}\right)$. It resulted respectively in 24 and 188 fibers and $160 N$ and $1254 N$ of $F_{0}^{m}$. The proposed methods were implemented in SOFA, an open source framework written in $\mathrm{C}++[8]$. Our implementation is not parallel and runs at approximately 15 frames per second on a standard PC, with an implicit Euler time integration scheme and MinRes iterative numerical solver (50 iterations maximum with a precision of $10^{-8}$ ), to integrate Newton equations. Unknowns are FEM node positions and velocities. Computational time is impacted by both the number of FEM nodes and fibers.

\subsection{Comparisons}

Fig. 3 shows a comparison of single fiber geometries (blue) and our multi fibers volumetric model embedded into two different FEM grid resolutions. The muscles were activated in concentric contraction manner while both ends of the tendon are fixed. The activation is generated from EMG measurements using the typical procedure in biomechanics research [9], and is sent at each time step to the Hill contractile element model to generate force. $L c 0$ optimal fiber length was automatically set as initial fiber length, thus, each fiber in all the studies at $t_{0}$ is considered as in its optimal fiber length. In parallel fiber configuration, as the fiber distribution is symmetrical around the center axis, total forces produced by the multi-fiber and wire models are exactly the same. In pennate and bipennate cases, the increased origin-insertion surface allows to arrange larger number of fibers by keeping same muscle fiber density. It results in higher longitudinal force even if there is a pennation angle. Multi-fiber models can account for non uniform pennation angles and fiber strains, resulting in significantly different 
total muscle forces. Results obtained using coarse (18 elements) and fine (608 elements) FEM grids closely match, showing that we can generate consistent forces regardless of FEM resolution choice.

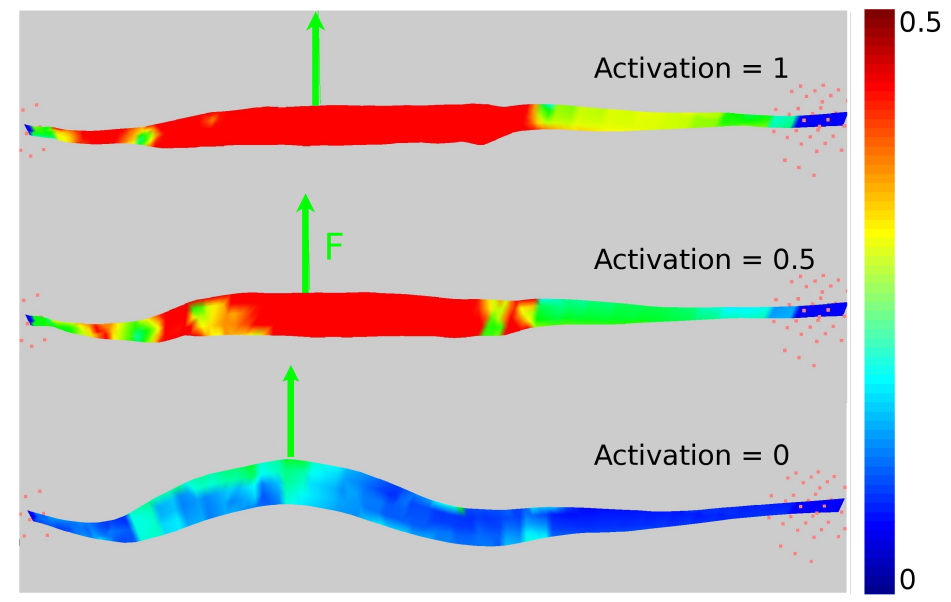

Fig. 4. Rectus femoris deformations during pulling in the transversal direction of the line of muscle force action, for activation levels of respectively $0,0.5$ and 1 . Color bar shows the maximum principal strain on a sagittal plane inside the muscle. The stiffness in transversal direction increased according to muscle contraction.

\subsection{Subject-specific Simulation}

Combined with registration forces, our typical deformable model can be easily personalized, by defining a surface mesh of the rectus femoris muscle obtained by manual MRI segmentation, as the target. Closest-point spring forces are applied to the surface points of the generic model. These forces are automatically propagated to the FEM nodes. By increasing the stiffness of these springs during the registration, data attraction is privileged, allowing an accurate coarse-tofine alignment of generic and target surfaces (given a sufficient number of FEM nodes). All the internal features (fibers and aponeurosis surface) are automatically estimated, thanks to the barycentric embedding. The personalized rectus femoris bipennate model is studied in isometric contraction, by varying the activation to $0,0.5$ and 1 , and pulling the muscle transversally to the line of muscle action by a constant force. The simulation shows at the equilibrium state, that the stiffness in transversal direction increased by increasing activation level (see Fig. 4). These models allows analyzing both longitudinal and transversal mechanical variable changes during muscle contraction. Simulations video link ${ }^{1}$

\footnotetext{
${ }^{1}$ http://www.lirmm.fr/ hayashibe/Miccai2014/miccaiSohusim.avi
} 


\section{Conclusion and Future Work}

We present a multi decoupled modeling method to simulate $3 \mathrm{D}$ muscles with complex architecture in real-time (15 FPS), that is a compromise between highly detailed 3D FEM and 1D Hill-type existing approaches. This leads to higher flexibility on the modeling side (separate thus optimized discretizations of active, isotropic and anisotropic parts). As expected, uniaxial simulations fit validated 1D Hill wire-segments behavior. Fast computation time allows to run the simulation in real-time using activation input based on real EMG measurements. We plan to validate this method with an extended set of subject-specific muscles reconstructed from MRI, and combine it with a skeletal model, to achieve comprehensive musculoskeletal simulations. We currently work on validating deformations using MRI data showing the knee in various postures combined with EMG measurements.

Acknowledgments. This work was supported by French ANR SoHuSim project.

\section{References}

1. Hoy, M. G., Zajac, F. E., and Gordon, M. E. A musculoskeletal model of the human lower extremity: The effect of muscle, tendon, and moment arm on the moment angle relationship of musculotendon actuators at the hip, knee, and ankle. J. Biomech, 23(2):157-169, 1990.

2. Delp, S. L., Anderson, F. C., Arnold, A. S., Loan, P., Habib, A., John, C. T., Guendelman, E., and Thelen, D. G. OpenSim: open-source software to create and analyze dynamic simulations of movement. IEEE trans. on bio-medical engineering, 54(11):1940-1950, 2007.

3. Blemker, S. S. and Delp, S. L. Three-Dimensional Representation of Complex Muscle Architectures and Geometries. Annals of Biomed. Eng., 33(5):661-673, 2005.

4. Johansson, T., Meier, P. and Blickhan, R. A finite-element model for the mechanical analysis of skeletal muscles. J. of theoretical biology, 206(1):131-149, 2000.

5. Oomens, C. W. J., Maenhout, M., van Oijen, C. H., Drost, M. R. and Baaijens, F. P. Finite element modelling of contracting skeletal muscle. Philosophical trans. of the Royal Society of London., 358(1437):1453-1460, 2003.

6. Blemker, S. S., Pinsky, P. M. and Delp, S. L. A 3D model of muscle reveals the causes of nonuniform strains in the biceps brachii. J. biomech., 38(4):657-665, 2005.

7. John a Hodgson, Sheng-Wei Chi, Judy P Yang, Jiun-Shyan Chen, Victor R Edgerton, and Shantanu Sinha. Finite element modeling of passive material influence on the deformation and force output of skeletal muscle. $J$. of the mechanical behavior of biomedical materials, 9:163-183, 2012.

8. Faure, F., Duriez, C., Delingette, H., Allard, J., Gilles, B., Marchesseau, S., Talbot, H., Courtecuisse, H., Bousquet, G., Peterlik, I. and Cotin S. SOFA: A Multi-Model Framework for Interactive Physical Simulation. Soft Tissue Biomechanical Modeling for Computer Assisted Surgery, 11:283-321, 2012.

9. Mitsuhiro Hayashibe and David Guiraud. Voluntary EMG-to-force estimation with a multi-scale physiological muscle model. Biomedical eng. online, 12(1):86, 2013. 